\title{
Analysis on Digital Image Processing for Plant Health Monitoring
}

\author{
${ }^{1}$ Alina Granwehr and ${ }^{2}$ Verena Hofer \\ 1,2 University Mohamed Khider Biskra, Biskra 07000, Algeria \\ 1alinagranwehr@protonmail.com
}

\author{
Article Info \\ Journal of Computing and Natural Science (http://anapub.co.ke/journals/jens/jens.html) \\ Doi: https://doi.org/10.53759/181X/JCNS202101002 \\ Received 16 October 2020; Revised form 26 November 2020; Accepted 25 December 2020. \\ Available online 05 January 2021. \\ (C)2021 Published by AnaPub Publications.
}

\begin{abstract}
The country's ability to become self-sufficient in food production is becoming increasingly important. Agriculture is the primary occupation of a large portion of the population in equatorial countries like India, where the climate is ideal for the spread of plants. Pests and diseases are in control of about $25 \%$ of crop loss, according to a recent study released by the Food and Agriculture Organization. Black spot, leaf spot, rust, mildew, and botrytis blight are the most common plant diseases. Deep learning is a relatively new research technique for image processing and pattern recognition that has been proven to be highly productive in detection of plant leaf diseases.
\end{abstract}

Keywords - Digital Image Processing, Convolutional Neural Network, Segmentation, Pathology

\section{INTRODUCTION}

Plant nutrition is the analysis of the chemical elements and compounds that are needed for plant growth, metabolism, and external supply. Plants' normal life cycles are disrupted because of nutrient deficiency. Mineral nutrients are divided into the following categories: micronutrients/trace minerals and macronutrients. Crop diseases can result in a significant reduction in net agricultural productivity [1]. Plant pathology is the study of biotic and abiotic factors that influence a plant's overall health. Farmers must diagnose the problem early to take sufficient and prompt action to prevent further losses. Farmers will be able to take proactive preventative steps because of this. Plant disease can be caused by living organisms such as insects, fungi, and bacteria, as well as non-living factors such as nutrient imbalances, drought or excessive soil moisture, insufficient light, low oxygen availability, and so on.

Current techniques are limited to diagnosing only macronutrient deficiencies, and the classification systems used for each plant are vastly different [2]. Only methods in which leaves were infected by diseases are included in the report. On the leaves, disease signs must be evident. Plant disease detection is a critical step in avoiding crop losses in terms of both quality and quantity in the agricultural production system. The manual plant disease monitoring method necessitates more processing time as well as plant disease knowledge. Since image processing techniques are quick, automatic, and precise, they are used for the detection, quantification, and identification of plant diseases. The key steps in disease detection using image processing techniques are image acquisition, image pre-processing, image segmentation, feature extraction, and disease recognition.

\section{A. Research Methodology}

Crop diseases and nutrient shortages can be detected using image processing. Image acquisition, pre-processing, segmentation, and feature extraction are all stages in image processing.

\section{B. Image Acquisition}

The first phase in the processing of images obtained from various sources. UAVs (unmanned aerial vehicles), drones, conveyors on the ground, cell phones, and cameras of various resolutions can all be used to capture the input images manually or automatically.

500 photographs taken directly from the farm were used to build a database of diseased and stable pomegranate plants. The neural network was also trained to classify the images into categories such as Good Fruit, Fruit Spot, Bacterial Blight, Fruit Rot, Good Leaf, and Leaf Spot. [2].

The output of a deep-learning-based classification system for identifying banana leaf diseases was tested in a series of experiments. The real data came from the Plant Village project, which is open-source and freely accessible 
on the internet. The entire dataset of 3700 images was annotated as safe (1643 images), black Sigatoka (240 images), and other groups.[3].

Images taken in the experimental fields of the International Institute of Tropical Agriculture (IITA) in Bagamoyo District, Tanzania, were used to create the dataset for detecting cassava leaf diseases. The dataset, which included 2,415 cassava leaf images for pronounced symptoms of each class, as well as an additional nutrient deficiency class of 336 images, was fine-tuned using the transfer learning process. [4].

\section{Leaf Annotation}

Massimo Minervini et al. [5] have created a method that allows for faster annotation of leaves in images derived from plant phenotyping experiments. To reduce the amount of user input, all pixels in the input image are labelled with an integer indicating which leaf they belong to, and graph-based interactive segmentation is used. The software tool and its source code are freely accessible online (as a Matlab programme, which is also described in the Plant Image Analysis database) and can be used with iPlant, a web and cloud-based plant biology infrastructure.

\section{LITERATURE SURVEY}

\section{A. Leaf Diseases Classification using Imaging Data}

The [6] suggested a new method for diagnosing 13 different leaf diseases by looking at them. The experiment database includes 3,000 original images culled from various internet sites, as well as a supplemented collection of 30,000 images. A series of steps followed, including image pre-processing, augmentation, deep CNN preparation, and fine-tuning. The result was that the augmentation process had a greater impact than fine-tuning in achieving a 96.3 percent overall accuracy of the qualified model. In [7] proposed a method for identifying and classifying banana diseases based on convolutional neural networks. The model is fed the pictures taken by the farmer. The key contribution is the use of deep neural networks to detect two well-known banana diseases, banana Sigatoka and banana speckle, in real-world scenarios and under difficult conditions. The method's applicability was tested using both RGB and grayscale images. Each image in the dataset was resized to $60 \times 60$ pixels and converted to grayscale during the pre-processing stage. The self-taught feature extraction model and the classification model are the two parts of the LeNet model.

The [8] developed computer software that uses digital image processing techniques to predict seed image for a seed lot. The proposed method reduces the amount of time it takes to calculate the seedling growth rate while also improving the precision of the measurements. Seeds were weighed, and pure seeds, other seeds, and inert matter were divided into different components using forceps and a thin ruler. Finally, the percentages of each component were determined after each component was weighed.

\section{B. Nutrient Deficiency Symptoms}

[9] presented a study of a digital image processing-based diagnostic method for nutrient deficiency in plants. The parameters included leaf area calculation, segmentation of the leaf's edge and veins, and extraction of features such as the leaf's form, mineral content, age, and colour.

Mu-loc is a machine-learning system for predicting mitochondrially localised proteins in plants developed [10]. They compiled a detailed dataset of Arabidopsis thaliana and Solanum tuberosum plant subcellular localization for the entire proteome. Amino acid structure, protein location weight matrix, and gene coexpression details were among the features extracted. They used SVM and DNN, two types of machine-learning methods, in the feature selection and model training process.

\section{DEEP LEARNING}

\section{Dataset Used}

Land images are gathered and submitted to a central repository. They're categorised, annotated, and organised into a database. There are several open-source databases available on the web that can be used for testing and experimentation. The tools required for classifier training and testing can be downloaded from any of the sources and fed into the classifier. Plant Village is a Penn State University unit dedicated to the research and development of technologies that can help farmers. It is sponsored by the global union of agricultural experts and offers an opensource forum for exchanging crop-growth expertise.

The group may use the UCI machine learning repository, which is hosted by the University of California, Irvine, to train models to perform different acts. Plant Village's disease classification challenge gathers codes written in a variety of programming languages and frameworks by a large number of users. The codes are put through their paces on a pre-existing dataset, and their accuracy is graded. They also issued a variety of docker containers for submitting codes, including Caffe, Tensor flow, Torch7, ScikitLearn, Octave, and Keras. 
Kaggle is an online platform that houses an $87 \mathrm{~K}$ RGB image dataset for various safe and unhealthy crop leaves that are divided into 38 different groups. The initial dataset was downloaded from GitHub and recreated using offline augmentation. The American Phyto pathological society's online image database contains thousands of scientifically peer-reviewed photographs of diseased, nutritionally impaired, pestaffected crops and plants. They are published in journals and are useful for plant pathology training, identification, and diagnosis. Any picture in the database is marked with information such as the host, types of causal agents, disorder, contributor, and collection/publication.

The Center for Open Science, a non-profit technology organization headquartered in Charlottesville, Virginia, owns the OSF (Open Science Framework), a free forum that promotes research and facilitates collaboration. They have the largest publicly accessible image collection for a single plant disease. The repository is exclusively for Northern Leaf Blight (NLB), a widespread and devastating disease of maize. It consists of 18,222 photographs taken in the field and 105,735 annotations performed manually by experts to mark lesions. The aim is to improve rapid image-based screening for NLB in order to train CNNs on images with annotated lesions and achieve a 96.7 percent final accuracy.

\section{Training the Dataset}

In the work [11] suggested an approach that was evaluated on five different types of crops and three different types of crop diseases. Collecting the dataset, pre-processing the dataset, training the Convolutional Neural Network (CNN) model to recognize the type of crop, training the CNN model to detect the disease and validation of model through obtained results are all steps in the implementation process. The InceptionV 3 model outperforms the Mobile Net model in terms of accuracy and validity loss, according to the findings.

Using deep learning, [12] investigated plant disease recognition from individual lesions and spots. It is extremely difficult to classify plant diseases using digital images. Deep learning methods, specifically CNNs, seem to be capable of solving the majority of the technological difficulties associated with plant disease classification. On the other hand, dataset limitations in terms of sample number and variety continue to stymie the creation of genuinely robust plant disease classification systems. While some attempts are being made to create more representative databases, and data sharing is becoming more popular, the amount of data available remains small. Since the inherent variability within each image is indirectly taken into account by the subdivision into smaller regions, the approach suggested in this article will not only increase the size of image datasets but also increase their diversity. This method has some flaws as well, but it produces more accurate results in the context of restricted data.

\section{Nine-Layer Convolutional Neural Network}

For plant disease detection, established a nine-layer deep CNN. Using leaf photos, the proposed Deep CNN model will effectively distinguish 38 different classes of healthy and diseased plants. In addition, the data augmentation boosts the number of training records from 49,598 to 55,636. An augmented dataset with 61,486 images and 3000 training epochs was used to train and test the most effective Deep CNN model. A total of 39 different groups of plant leaves and background images were used to train the deep CNN model. Image flipping, gamma correction, noise injection, principal component analysis (PCA) colour augmentation, rotation, and scaling were among the data augmentation techniques used. In the classification process, the overall average accuracy was 96.4 percent. The amount of training epochs, batch size, and dropout all had a bigger impact on the outcomes. The max-pooling approach outperforms average pooling by a wide margin. The proposed Deep CNN model outperforms other machine learning models in terms of predictive capacity and efficiency. Furthermore, the proposed model's accuracy and reliability was assessed using AUC -ROC curves, Precisions, Recalls, and F1 Scores.

\section{Pre-Trained Models}

The identification and classification of various fruit diseases using correlation coefficients and deep features (CCDF). The proposed method includes two main steps for detecting infected areas, followed by feature extraction and classification. A hybrid approach is used to improve the contrast of the input image in the first step. The correlation coefficient-based segmentation process, which separates the infected regions from the context, is the next step. Two deep pre-trained models (VGG16, Caffe Alex Net) are used in the second stage to extract features of selected diseases. Before the max-pooling stage, a parallel features fusion step is included to merge the extracted features. The genetic algorithm is used to pick the most discriminant features before moving on to the multi-class SVM for the final stage of classification. The proposed model achieved a 98.60 percent accuracy rate after being tested in the CASC-IFW and plant village datasets.

To perform symptom-wise identification of cucumber diseases, proposed a deep convolutional neural network model. An image segmentation approach that introduced powerful discrimination of disease symptoms and clutter context was used to segment the symptom images from cucumber leaf images collected in field conditions. A dataset containing four diseases, including anthracnose, downy mildew, powdery mildew, and target leaf spots, was created using the symptom images, and then augmented using the data augmentation process. Quantitative tests showed that the DCNN could achieve excellent recognition performance. The DCNN had 93.4 percent and 92.2 percent accuracy on the unbalanced and balanced datasets, respectively. Due to its rich feature presentations, AlexNet outperformed the DCNN in comparison tests. Both the DCNN and AlexNet performed better than the traditional classifiers. A 
study evaluating the efficiency of a CNN model deployed offline in real-time on a mobile device to detect cassava pests and diseases foliar symptoms.

\section{CONCLUSION}

Digital image processing techniques and deep learning models can be combined to create a framework for nutrient deficiency identification and early disease diagnosis. Future work will include developing an interactive technology framework for farmers to help them achieve high yields, as well as offering a single interface for all plant groups. Only a few works exist for diagnosing micronutrient deficiency symptoms that also cause serious damage to the plant/development. As a result, future research will focus on diagnosing micronutrient deficiencies. For efficient disease classification, color image processing can be used instead of a gray-scale image. The next stage of the CNN model will provide solutions for improving the health of the diseased plant based on the findings of plant pathology diagnosis.

\section{References}

[1]. L. Florea and C. Florea, "Directed color transfer for low-light image enhancement,” Digital Signal Processing, vol. 93, pp. 1-12, Oct. 2019.

[2]. G. Li, X. Shen, J. Li, and J. Wang, "Diagonal-kernel convolutional neural networks for image classification,” Digital Signal Processing, vol. 108 , p. 102898 , Jan. 2021

[3]. C. Wu and Z. Kang, "Robust entropy-based symmetric regularized picture fuzzy clustering for image segmentation," Digital Signal Processing, vol. 110, p. 102905, Mar. 2021.

[4]. D. Béréziat and I. Herlin, "Motion and acceleration from image assimilation with evolution models," Digital Signal Processing, vol. 83, pp. $45-58$, Dec. 2018

[5]. X. Wu and C.-N. Yang, "Partial reversible AMBTC-based secret image sharing with steganography," Digital Signal Processing, vol. 93, pp. 22-33, Oct. 2019

[6]. W. Wang, J. Qin, Y. Zhang, D. Deng, S. Yu, Y. Zhang, and Y. Liu, "TNNL: A novel image dimensionality reduction method for face image recognition," Digital Signal Processing, vol. 115, p. 103082, Aug. 2021.

[7]. D. Liu, Q. Su, Z. Yuan, and X. Zhang, "A blind color digital image watermarking method based on image correction and eigenvalue decomposition," Signal Processing: Image Communication, vol. 95, p. 116292, Jul. 2021

[8]. L. Ma, "Research on distance education image correction based on digital image processing technology," EURASIP Journal on Image and Video Processing, vol. 2019, no. 1, Jan. 2019.

[9]. L. Zhang, L. Zhang, and L. Zhang, "Application research of digital media image processing technology based on wavelet transform," EURASIP Journal on Image and Video Processing, vol. 2018, no. 1, Dec. 2018.

[10]. Z. Che and X. Zhuang, "Digital Affine Shear Filter Banks With 2-Layer Structure and Their Applications in Image Processing," IEEE Transactions on Image Processing, vol. 27, no. 8, pp. 3931-3941, Aug. 2018

[11]. M. Fan and H. Wang, "An enhanced fragile watermarking scheme to digital image protection and self-recovery," Signal Processing: Image Communication, vol. 66, pp. 19-29, Aug. 2018.

[12]. Y.-S. Lee, Y.-H. Seo, and D.-W. Kim, "Digital blind watermarking based on depth variation prediction map and DWT for DIBR freeviewpoint image," Signal Processing: Image Communication, vol. 70, pp. 104-113, Feb. 2019. 